\title{
Floods of July and September 1998 in Clark County, Nevada
}

\section{Background}

A series of severe thunderstorms swept through southern Nevada July 20-23 and September 11, 1998. Precipitation associated with these storms resulted in flooding in parts of Las Vegas Valley and the surrounding area within Clark County, Nev. (Fig. 1).

Clark County generally is drained by the Las Vegas Wash, Muddy River, and Virgin River systems. Las Vegas Valley is drained by Duck Creek, Flamingo Wash, Las Vegas Wash, and several smaller tributaries. Water in these drainages generally flows eastward through Las Vegas to Las Vegas Wash and on toward Lake Mead, an impoundment of the Colorado River. Muddy River flows southeast through Moapa Valley into the Overton Arm of Lake Mead.

\section{Data Collection}

The U.S. Geological Survey operates a network of streamflow-gaging stations in Clark County in cooperation with Federal, State, and local agencies. Data from these stations are crucial for water-supply planning, flood monitoring, emergency response, dam and reservoir-system operation, establishing floodinsurance rates, and engineering and maintenance of bridges, roads, and other structures.

Most of these stations provide real-time data through satellite relay or radiotelemetry. Data from these stations are used by the National Weather Service, the Clark County Regional Flood Control District, and other agencies to maintain water supplies, forecast floods, and issue flood warnings.

U.S. Geological Survey field crews obtained some of the highest discharge (flow) measurements ever recorded at several gaging stations during the July 20-23 and September 11, 1998, floods. Hydraulic surveys were made after the floods to determine peak discharge at stations where field crews were unable to obtain discharge measurements. These data contribute to understanding flood behavior, enhance efforts to minimize destruction caused by floods, and provide data for planning.

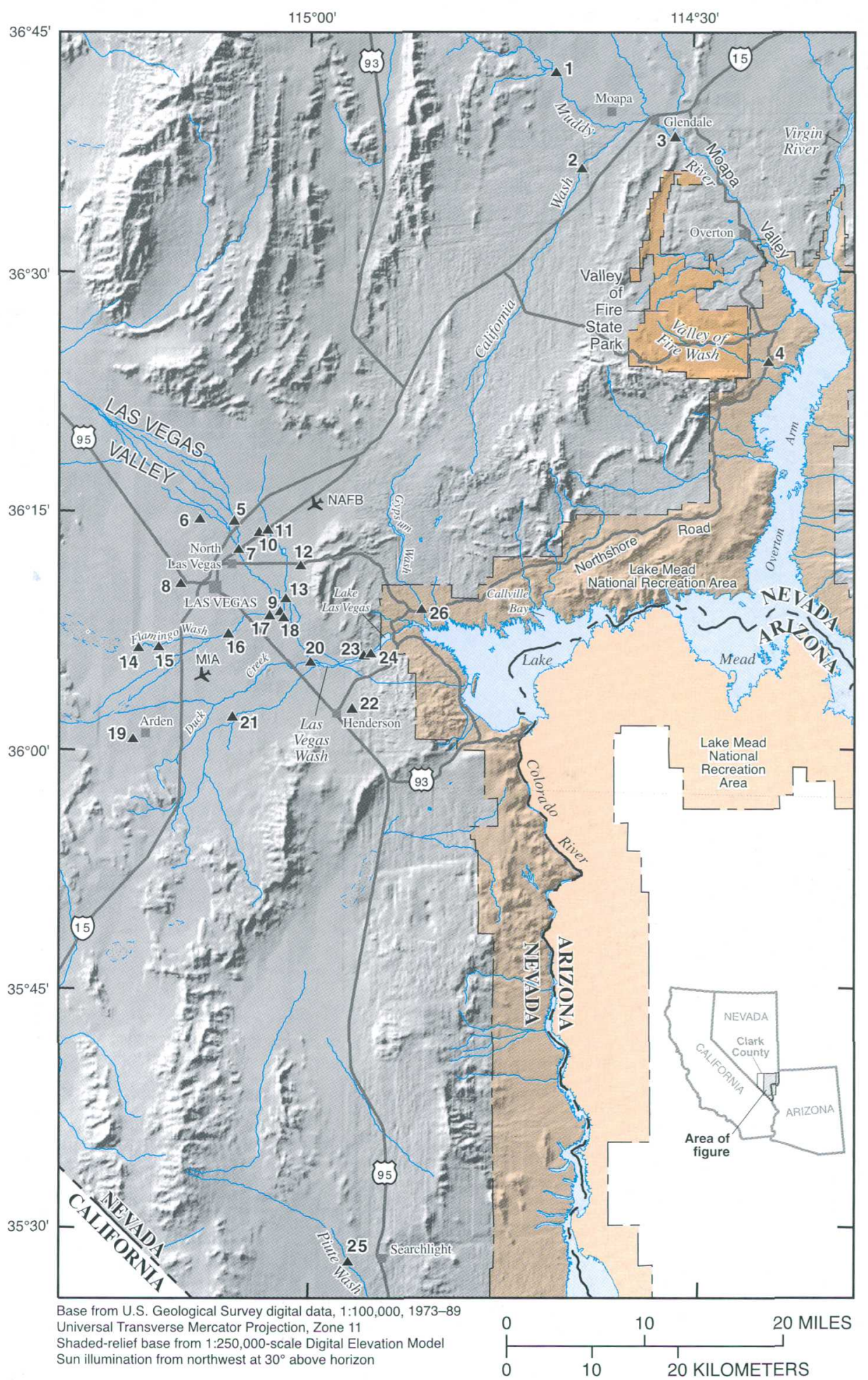

Figure 1. Geographic and hydrologic features in Clark County, Nev. Triangles locate streamflowgaging stations; labels indicate site numbers used in this report. MIA, McCarran International Airport; NAFB, Nellis Air Force Base. 


\section{Peak Discharges}

Information on peak discharges for selected gaging stations (Fig. 1) in Clark County, Nev., is shown in the table (below). The data include July and September 1998 peak discharges, gaging-station period of record, and date and magnitude of the largest historical peak discharge recorded prior to July 1998.

\section{Flood of July 1998}

During July.20-23, 1998, seyeral storms moved across Las Vegas Valley and other parts of Clark County. Lightning caused power outages and several fires. Numerous accidents and road closures were attributed to the heavy rain. Winds of more than 50 miles per hour were recorded at McCarran International Airport, and mudslides occurred along roadways. Thou- sands of residents were left without electricity and two deaths were attributed to these storms (Fink and Koch, 1998).

Rainfall in the Las Vegas Valley was recorded at 1 inch in 45 minutes by several rain gages in the early morning hours of July 20 , 1998 , as an intense thunderstorm cell moved southward across the valley. Another thunderstorm cell moved through Las Vegas Valley

\section{Peak discharges at selected sites in Clark County, Nev.}

[Site no.: Number used to identify gaging station in Fig. 1. Peak-discharge data are reported to differing accuracies depending on method of computation (such as whether instantaneousdischarge measurement or indirect computation) and on assessed data quality; data were compiled from U.S. Geological Survey (1961, 1976), Frisbie and others (1983, 1984), Bostic and others (1997), Bonner and others (1998), and Preissler and others (1999) and from unpublished data on file at U.S. Geological Survey offices in Las Vegas, Nev. -, not determined or not applicable]

\begin{tabular}{|c|c|c|c|c|c|c|}
\hline \multirow{2}{*}{$\begin{array}{l}\text { Site } \\
\text { no. }\end{array}$} & \multicolumn{2}{|r|}{ Streamflow-gaging station } & $\begin{array}{l}\text { Peak discharge } \\
\text { (cubic feet per second) }\end{array}$ & \multirow{2}{*}{$\begin{array}{l}\text { Period of record } \\
\text { through } 1998 \\
\text { (water years) }\end{array}$} & \multicolumn{2}{|c|}{$\begin{array}{l}\text { Largest historical peak } \\
\text { discharge recorded prior to } \\
\text { July } 20,1998\end{array}$} \\
\hline & Number & $\begin{array}{l}\text { July } 20-23 \\
1998\end{array}$ & $\begin{array}{l}\text { September } 11 \\
1998\end{array}$ & & Date & $\begin{array}{l}\text { Magnitude } \\
\text { (cubic feet } \\
\text { per second) }\end{array}$ \\
\hline 1 & 09416000 & Muddy River near Moapa, Nev. & 401 & $1913-18,1928-32,1944-98$ & August 16,1990 & 5,760 \\
\hline 2 & 09417300 & California Wash near Moapa, Nev. & 4,400 & $1981,1987-98$ & August 10,1981 & 30,600 \\
\hline 3 & 09419000 & Muddy River near Glendale, Nev. & 6,400 & $\begin{array}{c}1904-06,1910,1913-14 \\
1950-83,1984-98\end{array}$ & August 10, 1981 & 16,400 \\
\hline 4 & 09419545 & Valley of Fire Wash near Overton, Nev. & 200 & $1981,1987-98$ & August 10,1981 & 20,800 \\
\hline 5 & 09419649 & $\begin{array}{l}\text { North Las Vegas detention-basin outlet at Craig Road } \\
\text { near North Las Vegas, Nev. }\end{array}$ & 103 & $1992-98$ & August 9,1997 & 191 \\
\hline 6 & 094196497 & Gowan detention-basin outlet near North Las Vegas, Nev. & 238 & $1991-98$ & August 9,1997 & 644 \\
\hline 7 & 09419650 & Las Vegas Wash at North Las Vegas, Nev. & 740 & $1963-78,1982-98$ & July 3,1975 & 12,000 \\
\hline 8 & 094196557 & $\begin{array}{l}\text { Las Vegas creek at Meadows detention basin at Las } \\
\text { Vegas, Nev. }\end{array}$ & 23 & 1989-98 & July 15,1996 & 195 \\
\hline 9 & 09419658 & Las Vegas Wash near Sahara Avenue near Las Vegas, Nev. & 4,400 & $1975,1988-98$ & July 3,1975 & 4,350 \\
\hline 10 & 09419659 & $\begin{array}{l}\text { Sloan channel tributary at Las Vegas Boulevard near } \\
\text { North Las Vegas, Nev. }\end{array}$ & 920 & $1988-98$ & August 9,1997 & 260 \\
\hline 11 & 09419660 & Las Vegas Wash tributary near Nellis Air Force Base & 170 & $1961-84,1986-98$ & October 9,1972 & 618 \\
\hline 12 & 09419663 & Las Vegas Wash tributary south of Nellis Air Force Base & 600 & $1963-81,1983-98$ & September 4, 1963 & 296 \\
\hline 13 & 09419665 & $\begin{array}{l}\text { Sloan channel at Charleston Boulevard near Las Vegas, } \\
\text { Nev. }\end{array}$ & 1,230 & $1988-98$ & August 9,1997 & 700 \\
\hline 14 & 09419673 & $\begin{array}{l}\text { Flamingo Wash at Torrey Pines Drive near Las Vegas, } \\
\text { Nev. }\end{array}$ & 4.1 & $1988-98$ & July 16,1990 & 3,920 \\
\hline 15 & 09419674 & Flamingo Wash at Decatur Boulevard at Las Vegas, Nev. & 100 & $31966-81,1983-90,1992-98$ & August 10,1983 & 4,760 \\
\hline 16 & 094196775 & Flamingo Wash at Eastern Avenue near Las Vegas, Nev. & 809 & $4 \longdiv { 1 9 6 9 - 9 8 }$ & August 10,1983 & 4,700 \\
\hline 17 & 094196781 & Flamingo Wash at Nellis Boulevard near Las Vegas, Nev. $\quad 3,300$ & 1,900 & $1969-87,1989-98$ & June 10,1990 & 4,100 \\
\hline 18 & 094196783 & $\begin{array}{l}\text { Las Vegas Wash below Flamingo Wash confluence near } \\
\text { Las Vegas, Nev. }\end{array}$ & 6,100 & $1996-98$ & February 24,1998 & 4,450 \\
\hline 19 & 09419685 & Bird Spring wash near Arden, Nev. & - & $1987-98$ & May 2, 1997 & 27 \\
\hline 20 & 09419690 & Duck Creek at Whitney, Nev. & 3,000 & $1961-89,1998$ & August 30,1961 & 3,570 \\
\hline 21 & 09419695 & Pittman wash at Wigwam Parkway near Henderson, Nev. & 1,450 & $1988-98$ & August 10,1997 & 900 \\
\hline 22 & 09419740 & C-1 channel near Warm Springs Road at Henderson, Nev. & 53 & $1990-98$ & August 10,1997 & 2,700 \\
\hline 23 & 09419753 & $\begin{array}{l}\text { Las Vegas Wash above Three Kids wash below } \\
\text { Henderson, Nev. }\end{array}$ & 9,600 & $7 \longdiv { 1 9 5 7 - 9 8 }$ & August 14,1984 & 7,800 \\
\hline 24 & 09419756 & Las Vegas Wash overflow at Lake Las Vegas inlet & 9,560 & $1991-98$ & February 8,1993 & 2,620 \\
\hline 25 & 09423300 & Piute Wash tributary at Searchlight, Nev. & $+\infty$ & $1967-82,1984,1990,1998$ & August 13, 1982 & 400 \\
\hline 26 & - & Gypsum Wash near Callville Bay, Nev. & 17,000 & 1984 & July 22,1984 & 13,100 \\
\hline & $\begin{array}{l}{ }^{1} \text { Peak disch } \\
2 \text { Peak disch } \\
{ }^{3} \text { During } 196 \\
{ }^{4} \text { During } 196 \\
{ }^{5} \text { During } 196 \\
{ }^{6} \text { Peak disch } \\
7\end{array}$ & $\begin{array}{l}\text { eccurred July } 20,1998 . \\
-81 \text { and } 1984-85 \text {, equivalent station operated at Flamingo Wash at Las Vegas } \\
-89 \text {, equivalent station operated at Flamingo Wash at Maryland Parkway at L } \\
-87 \text {, equivalent station operated at Flamingo Wash near mouth at Las Vegas, } \\
\text { e occurred July } 23,1998 .\end{array}$ & $\begin{array}{l}\text { s, Nev. (09419675 } \\
\text { as Vegas, Nev. (0 } \\
\text { Nev. (09419678). }\end{array}$ & $94(9677)$ & $\therefore$ & \\
\hline
\end{tabular}


and northward across Clark County on the night of July 21, 1998. During this period, rainfall was reported at 1.14 inches at Valley of Fire State Park (Timothy E. Sutko, Clark County Regional Flood Control District, written commun., 1998).

The July 20, 1998, flooding caused by localized runoff was most severe in Flamingo Wash in Las Vegas Valley. The maximum discharge in Flamingo Wash increased from the western to the eastern part of the drainage area. Discharge peaked at Flamingo Wash at Eastern Avenue near Las Vegas (site 16, Fig. 1) at 4,100 cubic feet per second $\left(\mathrm{ft}^{3} / \mathrm{s}\right)$, the second highest flow on record, and then attenuated to 3,300 $\mathrm{ft}^{3} / \mathrm{s}$ at Nellis Boulevard near Las Vegas (site 17). The flood wave increased to $4,500 \mathrm{ft}^{3} / \mathrm{s}$ at Las Vegas Wash below Flamingo Wash confluence near Las Vegas (site 18). A maximum discharge of $2,650 \mathrm{ft}^{3} / \mathrm{s}$ was recorded at Las Vegas Wash above Three Kids wash below Henderson (site 23).

Several other storms crossed Clark County during the week of July 20, 1998. After causing flooding in Las Vegas Valley, the storm of July 21-22, 1998, moved northeastward across Valley of Fire Wash near Overton (site 4, Fig. 1), where peak streamflow discharge of 2,000 $\mathrm{ft}^{3} / \mathrm{s}$ was determined. This was the third highest peak flow on record for this site.

\section{Flood of September 1998}

A severe thunderstorm moving across Las Vegas Valley and northeastern Clark County on September 11, 1998, caused widespread damage and flooding. The storm produced high winds, hail, a tornado, and as much as 2 inches of rainfall in parts of Las Vegas Valley (Manning, 1998). More than 3 inches of rain fell in Moapa Valley, about 50 miles northeast of Las Vegas (Timothy E. Sutko, Clark County Regional Flood Control District, written commun., 1998). The Clark County Public Works Department estimated that Moapa Valley sustained damage to roadways amounting to approximately $\$ 400,000$.

Runoff from the September 11, 1998, storm occurred in most of the main drainage basins in Las Vegas Valley. Peak flows for the period of record occurred in Pittman wash (site 21, Fig. 1), Sloan channel (site 13), and other tributaries to Las Vegas Wash in Las Vegas Valley (sites 10 and 12). Peak flows were recorded at Las Vegas Wash near Sahara Avenue (site 9), below Flamingo Wash confluence (site 18, Fig. 2), above Three Kids wash (site 23), and at the overflow at Lake Las Vegas ${ }^{1}$ inlet (site 24).
Because the bypass conduit was closed for repairs, floodwaters (Fig. 3) passed into Lake Las Vegas ${ }^{1}$. The peak discharge of $9,600 \mathrm{ft}^{3} / \mathrm{s}$ at Las Vegas Wash above Three Kids wash was the highest recorded since 1957 (period of record) for this section of Las Vegas Wash. A peak flow of $6,550 \mathrm{ft}^{3} / \mathrm{s}$ (Steven Weber, Lake Las Vegas Resort ${ }^{1}$, oral commun., 1998) was released from Lake Las Vegas through a series of spillways back into Las Vegas Wash (Fig. 4).

The storm continued moving northeastward out of Las Vegas Valley and across Moapa Valley. The National Park Service estimated that along Northshore Road and in other parts of Lake Mead National Recreation Area, damage amounted to about $\$ 1,000,000$ as a result of flooding at Gypsum Wash (site 26, Fig. 1). The peak discharge of $401 \mathrm{ft}^{3} / \mathrm{s}$ at the streamflowgaging station Muddy River near Moapa (site 1) was the highest since 1993. The peak dis-

I"Lake Las Vegas" and "Lake Las Vegas Resort" are unofficial names in local use for a residentialresort development east-southeast of Las Vegas, Nev. Water flows into the lake from Las Vegas Wash and its tributaries during extreme flow events. charge of $6,400 \mathrm{ft}^{3} / \mathrm{s}$ was the third highest ever recorded at Muddy River near Glendale (site 3) and the highest since 1981. At California Wash near Moapa (site 2), the peak discharge of $4,400 \mathrm{ft}^{3} / \mathrm{s}$ was the highest since 1981 .

- Richard L. Kane and Jon W. Wilson

\section{References Cited}

Bonner, L.J., Elliott, P.E., Ectchemendy, L.P., and Swartwood, J.R.,1998, Water resources data, Nevada, water year 1997: U.S. Geological Survey Water-Data Report NV-97$1,636 \mathrm{p}$.

Bostic, R.E., Kane, R.L., Kipfer, K.M., and Johnson, A.W., 1997, Water resources data, Nevada, water year 1996: U.S. Geological Survey Water-Data Report NV-96-1, 611 p.

Fink, Jerry, and Koch, Edward, 1998, Summer storm thrashes Vegas: Las Vegas Sun, July 20, 1998, p. 1A.

Frisbie, H.R., LaCamera, R.J., Riek, M.M., Wood, D.B., 1983, Water resources data, Nevada, water year 1982: U.S. Geological Survey Water-Data Report NV-82-1, 360 p.

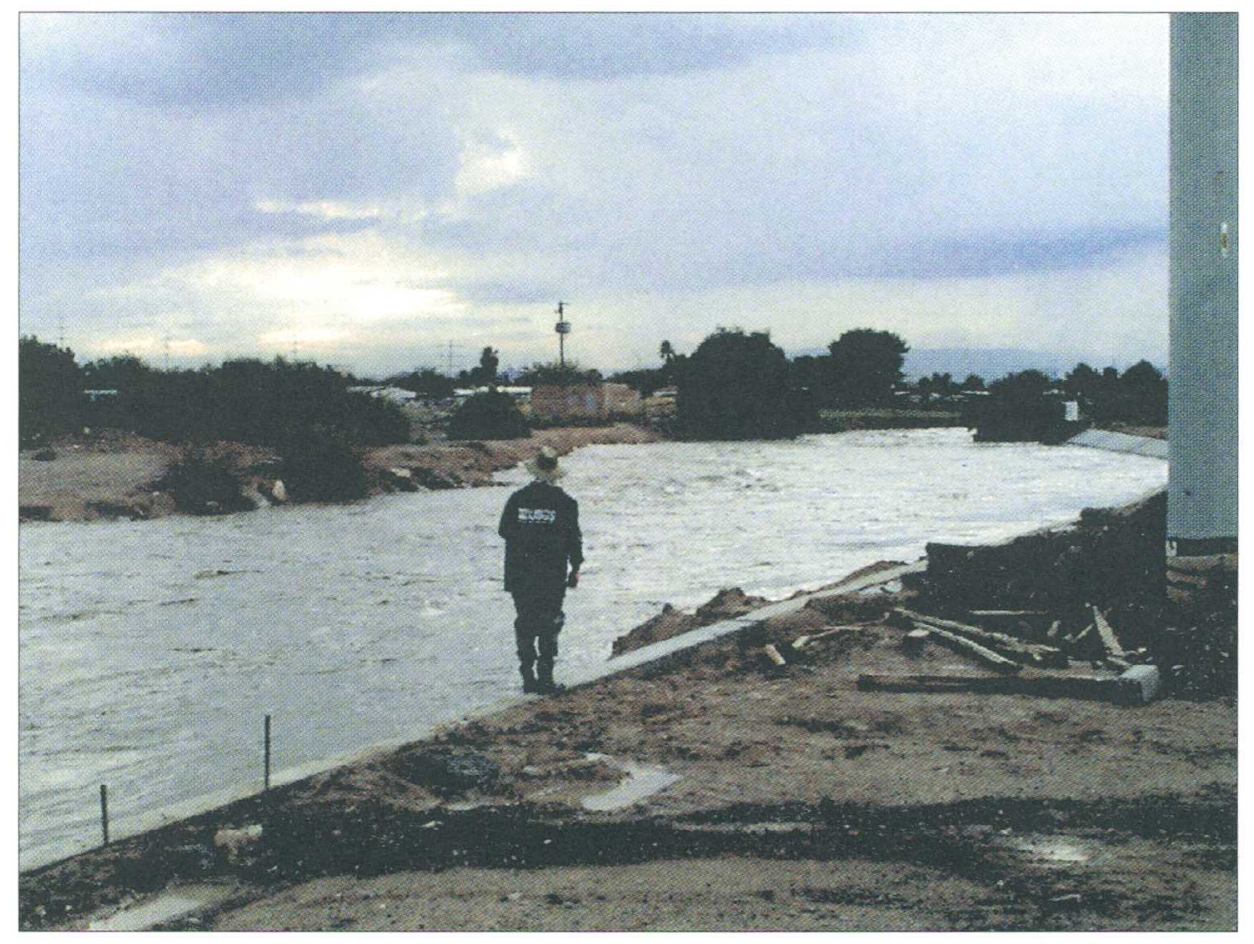

Figure 2. View upstream of flow at peak discharge $\left(6,100 \mathrm{ft}^{3} / \mathrm{s}\right)$ in Las Vegas Wash below Flamingo Wash confluence (site 18, Fig. 1 and table on facing page) during September 11, 1998, storm. 
-1984, Water resources data, Nevada, water year 1983: U.S. Geological Survey Water-Data Report NV-83-1, 328 p.

Manning, Mary, 1998, Officials still tallying damage from Friday's wild weather: Las Vegas Sun, September 15, 1998, p. 4A.
Preissler, A.M., Roach, G.A., Thomas, K.A., and Wilson, J.W., 1999, Water resources data, Nevada, water year 1998: U.S. Geological Survey Water-Data Report NV-98-1, 598 p.
United States Geological Survey, 1961, Surface water records of Nevada, 1961: U.S. Geological Survey, 118 p.

-1976, Water resources data for Nevada, water year 1975: U.S. Geological Survey Water-Data Report NV-75-1, 292 p.
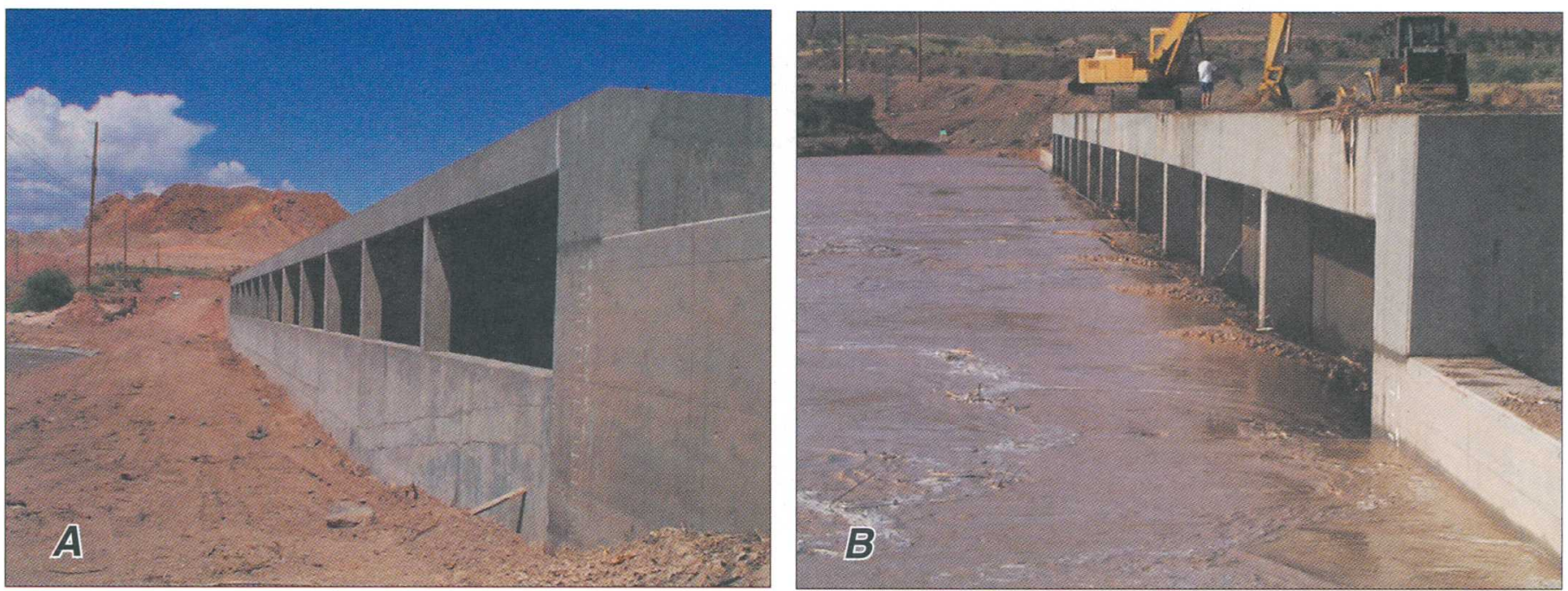

Figure 3. Views of Las Vegas Wash overflow at Lake Las Vegas inlet (site 24, Fig. 1 and table) and culvert structure. A, View when wash is dry. $B$, View during flood of September 11, 1998, showing floodwater discharging into Lake Las Vegas through culvert. Culvert openings are $8 \mathrm{ft}$ high; total width of structure is $240 \mathrm{ft}$. Photographs taken by G.C. Gortsema, U.S. Geological Survey.

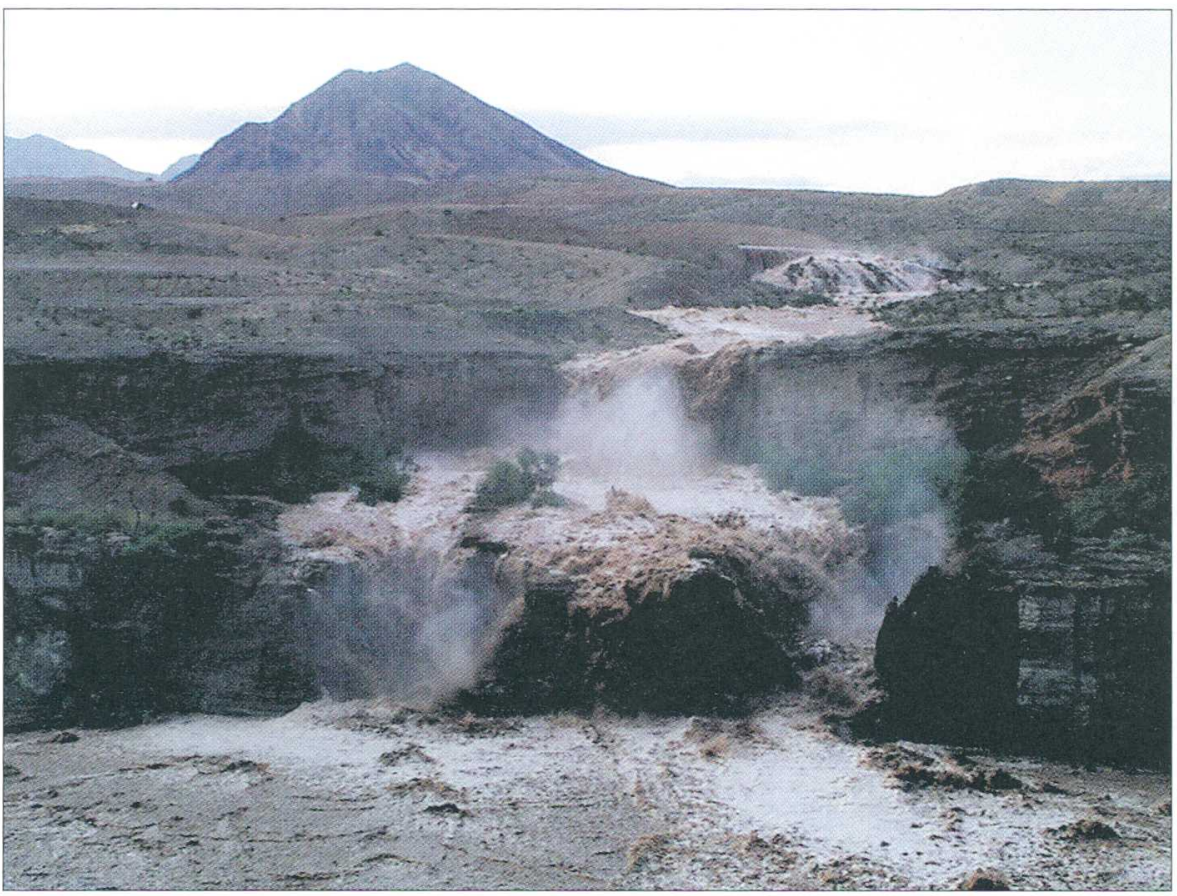

For more information on the U.S. Geological Survey studies described in this Fact Sheet, or on other aspects of water resources in Nevada, please contact:

Public Information Assistant U.S. Geological Survey 333 W. Nye Lane, Rm. 103 Carson City, NV 89706 telephone: (775) 887-7649 fax: (775) 887-7629

email: GS-W-NVpublic-info@usgs.gov URL: http://nevada.usgs.gov

Figure 4. Floodwaters released from one of three overflow spillways at Lake Las Vegas during flood of September 11, 1998, into Las Vegas Wash (below site 24; see Fig. 1 and table). Flow was estimated to be $6,000 \mathrm{ft}^{3} / \mathrm{s}$ at time photograph was taken. 